University of Nebraska - Lincoln

DigitalCommons@University of Nebraska - Lincoln

\title{
Two-Center Effect on Low-Energy Electron Emission in Collisions of 1-MeV/u Bare lons with Atomic Hydrogen, Molecular Hydrogen, and Helium. I. Atomic Hydrogen
}

\author{
Lokesh C. Tribedi \\ Tata Institute of Fundamental Research, Homi Bhabha Road, Colaba, Mumbai 400005, India \\ P. Richard \\ J.R. Macdonald Laboratory, Department of Physics, Kansas State University, Manhattan, Kansas \\ 66506-2601 \\ L. Gulya's \\ Institute of Nuclear Research of the Hungarian Academy of Science (ATOMKI), P.O. Box 51, H-4001 \\ Debrecen, Hungary \\ M. Eugene Rudd \\ University of Nebraska - Lincoln, erudd@unl.edu \\ R. Moshammer \\ Fakultat fur Physik, University of Freiburg, Freiburg 79104, Germany
}

Follow this and additional works at: https://digitalcommons.unl.edu/physicsrudd

Part of the Physics Commons

Tribedi, Lokesh C.; Richard, P.; Gulya's, L.; Rudd, M. Eugene; and Moshammer, R., "Two-Center Effect on Low-Energy Electron Emission in Collisions of 1-MeV/u Bare lons with Atomic Hydrogen, Molecular Hydrogen, and Helium. I. Atomic Hydrogen" (2001). M. Eugene Rudd Publications. 25.

https://digitalcommons.unl.edu/physicsrudd/25

This Article is brought to you for free and open access by the Research Papers in Physics and Astronomy at DigitalCommons@University of Nebraska - Lincoln. It has been accepted for inclusion in M. Eugene Rudd Publications by an authorized administrator of DigitalCommons@University of Nebraska - Lincoln. 


\title{
Two-center effect on low-energy electron emission in collisions of $1-\mathrm{MeV} / \mathrm{u}$ bare ions with atomic hydrogen, molecular hydrogen, and helium. I. Atomic hydrogen
}

\author{
Lokesh C. Tribedi, ${ }^{1, *}$ P. Richard,${ }^{2}$ L. Gulyás, ${ }^{3}$ M. E. Rudd, ${ }^{4}$ and R. Moshammer ${ }^{5}$ \\ ${ }^{1}$ Tata Institute of Fundamental Research, Homi Bhabha Road, Colaba, Mumbai 400005, India \\ ${ }^{2}$ J.R. Macdonald Laboratory, Department of Physics, Kansas State University, Manhattan, Kansas 66506-2601 \\ ${ }^{3}$ Institute of Nuclear Research of the Hungarian Academy of Science (ATOMKI), P.O. Box 51, H-4001 Debrecen, Hungary \\ ${ }^{4}$ Department of Physics and Astronomy, University of Nebraska, Lincoln, Nebraska 68588-0111 \\ ${ }^{5}$ Fakultat fur Physik, University of Freiburg, Freiburg 79104, Germany \\ (Received 25 September 2000; published 17 May 2001)
}

\begin{abstract}
We have investigated ionization mechanisms in fast ion-atom collisions by measuring the low-energy electron emission cross sections in a pure three-body collision involving bare carbon ions $(v=6.35$ a.u.) colliding with atomic hydrogen targets. The measurements have also been extended to molecular hydrogen and helium targets. In this paper we provide the energy and angular distributions of double differential cross sections of low-energy electron emission for atomic hydrogen targets. The Slevin rf source with a high degree of dissociation was used to produce the atomic $\mathrm{H}$ target. It is found that the two-center effect has a major influence on the observed large forward-backward angular asymmetry. A detailed comparison is presented with calculations based on the continuum distorted-wave (CDW) and CDW-EIS (eikonal initial-state) approximations. Both the continuum distorted-wave calculations provide a very good understanding of the data, whereas the first Born calculation predicts almost symmetric forward-backward distributions that do not agree with the data. The two-center effect is slightly better represented by the CDW calculations compared to the CDW-EIS calculation. The total cross sections are, however, in good agreement with the theories used. The results for molecular hydrogen and helium will be discussed in the following paper.
\end{abstract}

DOI: $10.1103 /$ PhysRevA.63.062723

PACS number(s): 34.50.Fa

\section{INTRODUCTION}

Ionization is one of the dominant inelastic processes in intermediate and fast ion-atom collisions. The ionization of atomic hydrogen by swift bare ions is one of the simplest atomic collision processes leading to pure three-body ionization and provides a most suitable testing ground of quantummechanical theoretical models that describe electron emission in the ionization process. Although there have been numerous experimental and theoretical studies on ion-impact ionization in the past, the understanding of the energy and angular distributions of low-energy electron emission in heavy ion-induced ionization is far from complete. Most of the previous experiments have been carried out using multielectron targets. The total ionization cross sections (TICSs) for atomic hydrogen targets have been measured in the past [1-3] using protons. However, TICSs result from an integration over the momenta of the three particles in the final state. They cannot provide the finer details of the process since these are obtained by integrating over the momenta of all three particles in the final state. In order to gain more insight into the ionization dynamics, one needs to measure the energy and angular distributions of the low-energy electrons since these electrons carry the bulk of the cross sections, although they are difficult to detect. The double differential ionization cross sections (DDCSs), differential in emission angle and energy for a pure three-body collision system, can provide stringent tests to the most sophisticated theories on ionization. Such measurements for atomic hydrogen have

*Email address: lokesh@tifr.res.in been carried out only for low charged projectiles such as electrons [4], protons [5,6], and helium ions [7].

The first measurement on electron DDCS in the ionization of atomic hydrogen by highly charged heavy ions has been reported only recently [8] at high velocity ( $v=10$ a.u.). The initial-state electron cloud is highly perturbed under the influence of heavy ions, and the ejected electron moves in the long-range Coulomb fields originating from the ionized target and the moving ion. Such a two-center effect modifies drastically the angular distributions of the emitted electrons causing forward-backward asymmetry, which again depends on the electron energy. Such two-center effects (TCEs), which are stronger for projectiles with higher atomic numbers $(Z)$ and lower velocity $(v)$, have been explored mostly for high velocity ions $(v \geqslant 10)[9]$ on He. To explore the angular distribution patterns of different energy electrons under the influence of the TCE, it is therefore necessary to measure the electron DDCS using lower velocity heavy ions. We present here the energy and angular distributions of electrons with energies between 1 and $300 \mathrm{eV}$ emitted in the ionization of atomic hydrogen in collisions with $1 \mathrm{MeV} / \mathrm{u}$ $(v=6.35$ a.u. $)$ bare carbon ions.

It is known that the two-center electron emission cannot be described by the first Born calculations. At intermediate and high energies, distorted wave theories provide an adequate framework to treat the electron emission process under heavy-ion impact. At present, two of them-the continuum distorted-wave (CDW) and continuum distortedwave with eikonal initial-state (CDW-EIS) approximations-have been studied in detail. The CDW-EIS approximation, developed by Crothers and McCann [10] and extended to multielectronic targets by Fainstein and coworkers $[11,12]$, has been successfully applied to explain the 
two-center effect on electron emission. The continuum distortion applied in the outgoing channel is shown to be adequate to describe the dynamics of the ionized electron in the combined Coulomb fields of the projectile and target. The CDW theory for ionization was introduced by Belkic [13]. However, the model received less interest due to the incorrect normalization of distorted waves resulting in overestimated cross-section values at low impact energies [14]. The significant difference between the CDW and CDW-EIS approximations lies in the forms of distortions applied in the initial channel. The former accounts for the distortion by using an eikonal phase, while the latter, similar to the final channel, uses a continuum distortion. The eikonal phase corresponds to the asymptotic behavior of the continuum distortion at asymptotic distances, consequently the two-center character of the distorted-wave functions is highly reduced. Moreover, recently it has been shown [15-17] that the use of more realistic wave functions, instead of $\mathrm{H}$-like wave functions, for the initial and final states of the electron, provides a better agreement with the data for multielectron targets. However, in the case of an atomic hydrogen target, where the initial wave function is known exactly, such modifications are not required.

It is known that in the case of two-electron or multielectron targets, the contributions from double electron or multielectron process electron-electron correlation can be substantial enough to complicate the data analysis and a comparison with theoretical methods suffers from ambiguity. In spite of reasonable success in describing the features of electron emission in fast ion atom collisions, the CDW-EIS calculations are shown to deviate from the measured data, especially in the extreme forward and backward angles for $\mathrm{He}$, $\mathrm{H}_{2}$, Ne, and even for atomic hydrogen targets.

Recoil-ion momentum spectroscopy (RIMS) using the cold-target technique has also been used recently to study the two-center effect and post-collision interactions and their influences on the emission of low-energy electrons and recoil ions [18]. The relation between electron spectroscopy and RIMS has also been addressed recently [19-21] in order to study the ion-atom ionization mechanism. The observed shift in the recoil-ion and electron longitudinal momentum distributions in the opposite directions is believed to be associated with such post-collision interactions (PCIs) [22], which are shown to be stronger with higher values of perturbation strength $S_{p}=Z_{p} / v_{p}\left(Z_{p}\right.$ and $v_{p}$ are the atomic number and velocity of the projectile). A large shift and hence a large PCI is observed for $S_{p}=2.0$, whereas a negligible shift in the electron and recoil-ion longitudinal distributions is noticed in the case of much smaller values of $S_{p}(=0.6)[19,20]$. However, in spite of a negligible shift in the momentum distributions [21,22], a large forward-backward asymmetry has been observed in the electron emission for $\mathrm{C}^{6+}+\mathrm{He}$ for which $S_{p}$ was quite small, e.g., 0.6 [23] and 0.4 [9]. The aim of the present measurement is to explore the two-center effect by measuring the forward-backward asymmetry in the angular distribution of low-energy electron emission in fast ion-atom collisions with atomic hydrogen with a perturbation strength of nearly $1.0\left(S_{z}=0.94\right)$.

\section{EXPERIMENTAL DETAILS AND DATA ANALYSIS}

The experimental technique has been described in our earlier letter communication [8] and therefore we give the necessary details only. The highly collimated energy and charge-state selected beam of $1 \mathrm{MeV} / u$ bare carbon ions was obtained from the tandem Van de Graaff accelerator of the J. R. Macdonald Laboratory at Kansas State University (KSU). The beam interacts with the atomic hydrogen jet target $2 \mathrm{~mm}$ below the nozzle. A commercially available (Leisk engineering) rf hydrogen atom source, developed by Slevin and Sterling [24], was fed with a high-purity hydrogen gas at a pressure of 0.3-0.4 torr. The outlet gas contains some undissociated $\mathrm{H}_{2}$ along with atomic hydrogen, which were allowed in the chamber through a 1-mm-diam capillary tube. The $x, y$, and $z$ positions of the nozzle could be adjusted in order to align the gas jet with respect to the beam to obtain a maximum dissociation fraction.

The experimental setup including the spectrometer was constructed at the University of Nebraska and moved to KSU for the earlier experiments and was also used in the present investigations. The collimator geometry for the beam entrance was changed slightly. A turbomolecular pump was used to evacuate the chamber to $1 \times 10^{-7}$ torr. The various tests for performance of the spectrometer are similar to those described in Refs. [5,6]. A $\mu$-metal shield was used inside the chamber to reduce the stray magnetic field. A currentcarrying coil placed in the horizontal plane around the chamber was enough to reduce the stray magnetic field below 5 $\mathrm{mG}$ in the region where the electrons travel before entering the analyzer. A hemispherical electrostatic analyzer $[5,6]$ made of oxygen-free high-conductivity copper with inner and outer radii of 25 and $35 \mathrm{~mm}$ was used. The spherical surfaces were coated with carbon soot to reduce secondary electron production from the copper surface due to the electron bombardment. Before entering the analyzer, the electrons had to pass through a collimator made of a copper tube with two rectangular grounded apertures, one on each end. These two apertures of widths 4 and $3 \mathrm{~mm}$ mainly define the effective path-length solid-angle integral (see below). Additional apertures at the entrance and the exit of the analyzer were biased with a small voltage $V_{0}$ in order to preaccelerate the electrons entering the analyzer. It was found that $V_{0}=$ $+5 \mathrm{~V}$ was enough to improve the collection efficiency of the low-energy electrons. The energy-analyzed electrons were detected by a channel electron multiplier (CEM) mounted on the exit of the analyzer. The cone of the CEM was biased at $+100 \mathrm{~V}$ to help the low-energy electrons reach the detector.

The spectrometer could be rotated between $15^{\circ}$ and $165^{\circ}$, and electrons with energies between 1 and $300 \mathrm{eV}$ were detected at various angles at intervals of $10^{\circ}$ or $15^{\circ}$. The electron spectrum was taken with the rf power on $\left(S^{\mathrm{on}}\right)$ and RF power off $\left(S^{\text {off }}\right)$, keeping the jet pressure the same (about 0.3 $\mathrm{T})$ in both cases. From these measurements, one can determine the ratio $(R)$ of ionization cross sections (i.e., DDCS) for $\mathrm{H}$ to that for $\mathrm{H}_{2}$ [see Eq. (2) below]. The absolute cross section $\left(\sigma_{2}\right)$ for $\mathrm{H}_{2}$ was determined using a static gas condition with the rf off. The cross sections $\left(\sigma_{1}\right)$ for $\mathrm{H}$ were obtained using these cross sections and the measured ratio 
TABLE I. Measured double differential cross sections (in units of $\mathrm{Mb} / \mathrm{eV}$ sr) for different angles. Typical errors are about $25-30 \%$ except for $\epsilon \leqslant 5 \mathrm{eV}$, for which the errors could be 40-45\%. For backward angles the uncertainty is large (about 40-45\%) for higher energy $(\geqslant 100 \mathrm{eV})$ electrons due to a substantial background.

\begin{tabular}{ccccccccc}
\hline \hline$\theta \backslash \epsilon(\mathrm{eV})$ & 1 & 3 & 5 & 10 & 40 & \multicolumn{1}{c}{100} & 200 & 240 \\
\hline $15^{\circ}$ & 3.15 & 2.50 & 3.1 & 1.69 & 0.233 & 0.0192 & 0.00113 & 0.00110 \\
$45^{\circ}$ & 3.56 & 2.96 & 3.3 & 3.660 & 0.312 & 0.0388 & 0.0101 & 0.0372 \\
$60^{\circ}$ & 4.26 & 3.19 & 2.63 & 2.94 & 0.465 & 0.090 & 0.064 & 0.030 \\
$70^{\circ}$ & 3.97 & 3.12 & 2.74 & 3.34 & 0.612 & 0.17 & 0.051 \\
$90^{\circ}$ & 3.09 & 2.64 & 2.48 & 2.26 & 0.306 & 0.037 & $3.09 \times 10^{-4}$ \\
$105^{\circ}$ & & 1.10 & 0.93 & 0.92 & 0.053 & 0.00227 & $7.036 \times 10^{-5}$ \\
$120^{\circ}$ & 0.85 & 0.946 & 0.61 & 0.382 & 0.0137 & $7.36 \times 10^{-4}$ & \\
$135^{\circ}$ & & 0.496 & 0.49 & 0.28 & 0.0073 & $6.428 \times 10^{-4}$ & $7.036 \times 10^{-4}$ & \\
$160^{\circ}$ & & 0.652 & 0.353 & 0.320 & 0.0060 & $3.93 \times \times^{-4}$ & \\
\hline \hline
\end{tabular}

$(R)$. The details of the measurements of $\sigma_{2}$ can be found in Paper II. In brief, the chamber was flooded with low pressure (0.1-0.3 mTorr) $\mathrm{H}_{2}$ gas. The low gas pressure was to minimize the scattering of low-energy electrons before entering the analyzer. For absolute normalization of the $\mathrm{H}_{2}$ data, we have measured the electron spectrum at different angles for 1.5 MeV $p+\mathrm{He}$, for which the absolute cross sections are known [25]. The normalization constant was independent of electron energy or angle within about $7 \%$ for $\varepsilon=1$ $-300 \mathrm{eV}$. The efficiency of the channel electron multiplier, which is included in the derived normalization, is known to remain constant $(\approx 0.95)$ [26,27] for electron energies between 0 and $500 \mathrm{eV}$ since the front cone of the CEM was biased to about $+100 \mathrm{~V}$. The electron DDCS for $\mathrm{H}$ was found from the following expression:

$$
R=\frac{\sigma_{1}}{\sigma_{2}}=\frac{1}{\sqrt{2} D_{f}}\left(\frac{S^{\text {on }}}{S^{\text {off }}}-1+D_{f}\right)
$$

where $S^{\text {on }}$ and $S^{\text {off }}$ are the background subtracted electron counts for the same number of incident ions for a given electron energy and angle. This equation was used before in Refs. [4] and [5]. The DDCS for $\mathrm{H}$ atoms is then given by $\sigma_{1}=R \sigma_{2}$. The typical uncertainty in $\sigma_{1}$, which is determined by Eq. (1), was $\sim 20-25 \%$ (see Table I).

Since the gas jet at the outlet of the rf source contains some amount of undissociated molecules along with the atomic hydrogen, it is necessary to find the dissociation fraction $D_{f}$ in order to derive $R$ from Eq. (1). The quantity $D_{f}$ was measured in situ using a method called the $9-\mathrm{eV}$ proton method [5]. The spectrum of recoil ions produced in the collision of bare ions with $\mathrm{H}_{2}$ contains a broad peak of $\mathrm{H}^{+}$ having an energy around $9 \mathrm{eV}$. Two protons each of energy $9 \mathrm{eV}$ are produced by the Coulomb explosion of a doubly ionized $\mathrm{H}_{2}$ molecule [28] due to the interaction with the passing projectile. These protons also arise from the dissociation of the $2 p \sigma_{u}$ and some other nearby states of $\mathrm{H}_{2}{ }^{+}$ [29], and the total energy released $(18 \mathrm{eV})$ is shared by the two protons. The protons are detected by the same electrostatic spectrometer used for the electron detection, by reversing the polarities of the potentials applied on the inner and outer hemispheres. The cone of the CEM was biased to a high positive voltage $(+2.2 \mathrm{kV})$ and the rear was grounded. The dissociation fraction $\left(D_{f}\right)$ is defined as

$$
D_{f}=1-\frac{S^{\text {on }}}{S^{\text {off }}}
$$

where $S^{\text {on }}$ and $S^{\text {off }}$ represent the yields of the $9 \mathrm{eV}$ protons detected with the rf power on and off, respectively. The yields are determined from the areas under the 9-eV peaks. The best spectrum is shown in Fig. 1. The highest dissociation fraction was $88 \%$. However, the typical dissociation fraction was found to be $\sim 80-85 \%$ and almost independent of angle except for extreme forward and backward angles for which the $D_{f}$ was somewhat lower ( $\left.\sim 70 \%\right)$. This could be due to geometrical effects and to the fact that the path lengths seen by the detector are largest for these angles. This observation is consistent with the results of Kerby et al. [6]. However, the dissociation fraction obtained in the present experiment is even larger than that obtained in the previous experiments using the same source $[6,8]$. We have also determined $D_{f}$ by a second method, which was based on binary

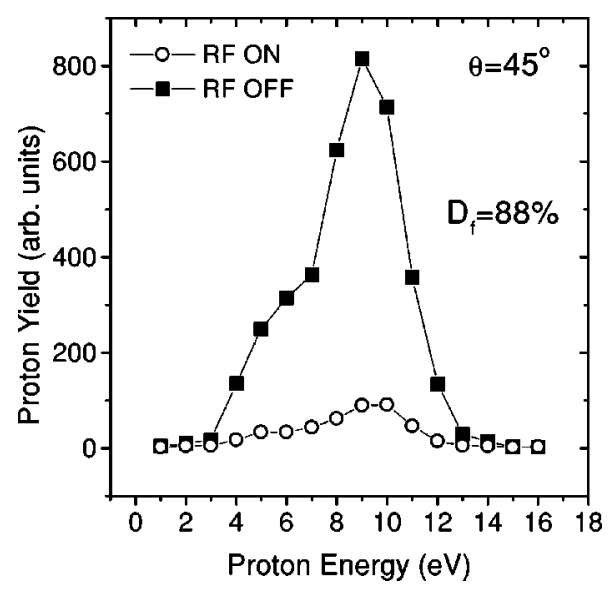

FIG. 1. (a) The spectrum showing the proton peak at $9 \mathrm{eV}$ produced in the collision $\mathrm{C}^{6+}+\mathrm{H}_{2}$ for $\theta=90^{\circ}$ for $\mathrm{RF}$ power on and off. 


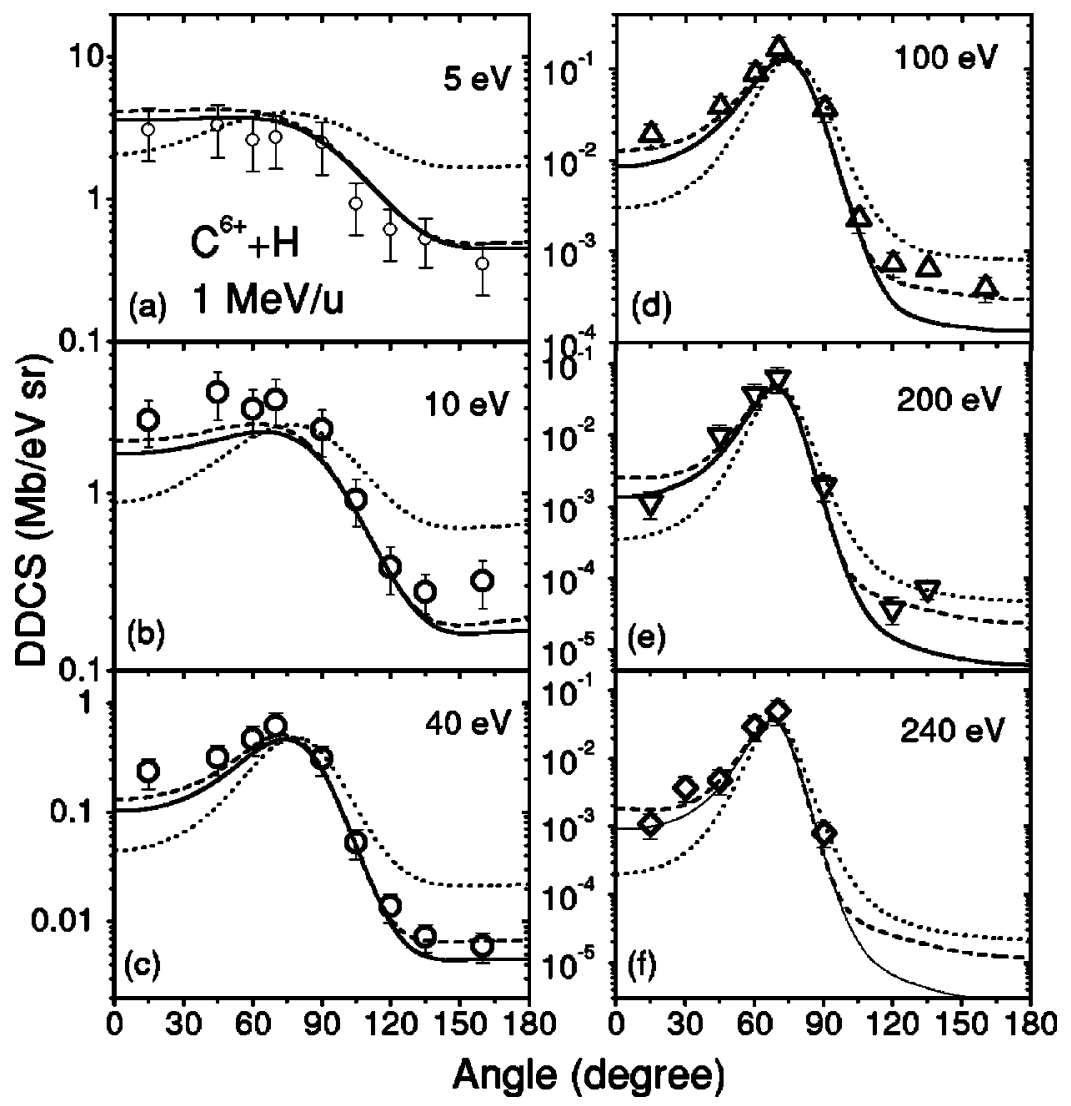

FIG. 2. (a) The angular distribution of electron DDCS for different energies. The solid, dashed, and dotted lines represent the CDW-EIS, $\mathrm{CDW}$, and $B 1$ calculations, respectively.

encounter electron yields. The $D_{f}$ value obtained with these two methods agrees within $\sim 5 \%$.

The energy dependence of the DDCS was studied for ten different angles between $15^{\circ}$ and $160^{\circ}$. At each angle, electrons having energies between 1 and $300 \mathrm{eV}$ were detected. We report the cross sections for atomic hydrogen in this first paper (paper I). We have also measured the angular distributions of low-energy electron DDCSs for the simplest twoelectron systems such as helium and molecular hydrogen, which are described in paper II.

\section{RESULTS AND DISCUSSIONS}

\section{A. Angular distributions of the DDCS}

The angular distributions of the electron DDCS for different electron energies are displayed in Fig. 2. Six different energies - 5, 10, 40, 100, 200, and $240 \mathrm{eV}$-were chosen to represent the behavior of the entire energy range investigated. For each energy, the angular distributions are compared with three theoretical models such as the First Born (B1), CDW-EIS, and CDW models. The angular distributions are found to have peaklike structures around $70^{\circ}$ only above $40 \mathrm{eV}$. At lower energies, the distributions show an almost flat behavior for the forward angles and a large decrease in the cross sections for backward angles. The peaks in the angular distributions, of which the positions follow the $\theta=\cos ^{-1}\left(v_{e} / 2 v_{p}\right)$ rule, are due to the binary collisions (commonly known as the binary encounter approximation or BEA) between the projectiles and electrons. The widths of the peaks are due to the initial momentum distributions of the electrons. It is well seen that this is the dominant process for ejecting medium- and high-energy electrons at the given ejection angle. For lower electron energies, other mechanisms, such as the dipole or distant collisions, become dominant in forming the angular distributions of the DDCS. However, similar to the BEA, this process also predicts a symmetric peak around $90^{\circ}$. This is the gross feature that has already been accounted for in the $B 1$ model. The results from the distorted wave theories show that the effects of the TCE appear mostly at the tails of the peaks by changing drastically the asymmetry character.

The $B 1$ calculations show a large deviation from the data in general, as far as the angular distributions are concerned. The shapes of the distributions are similar to the experimental data only above $40 \mathrm{eV}$. The calculations, in general, underestimate the data by a large factor for small forward angles and overestimate them at large backward angles [see Figs. 2(c)-2(f)]. The behavior in the forward angles can be understood in terms of the two-center effect, i.e., due to the long-range Coulomb fields arising from the residual recoil ion and projectile acting on the electrons. The electrons emitted between $60^{\circ}-90^{\circ}$ are mostly dominated by the binary collisions and are reproduced by the $B 1$ calculations.

The TCE causes a large enhancement in the forward direction and a depletion in the backward angles, an effect that is included in the continuum distorted-wave calculations. The CDW-EIS and CDW models reproduce the overall angular distributions quite well [solid and dashed lines in Figs. 2(a)-2(f)]. For example, at 40 and $100 \mathrm{eV}$, the CDW-EIS 


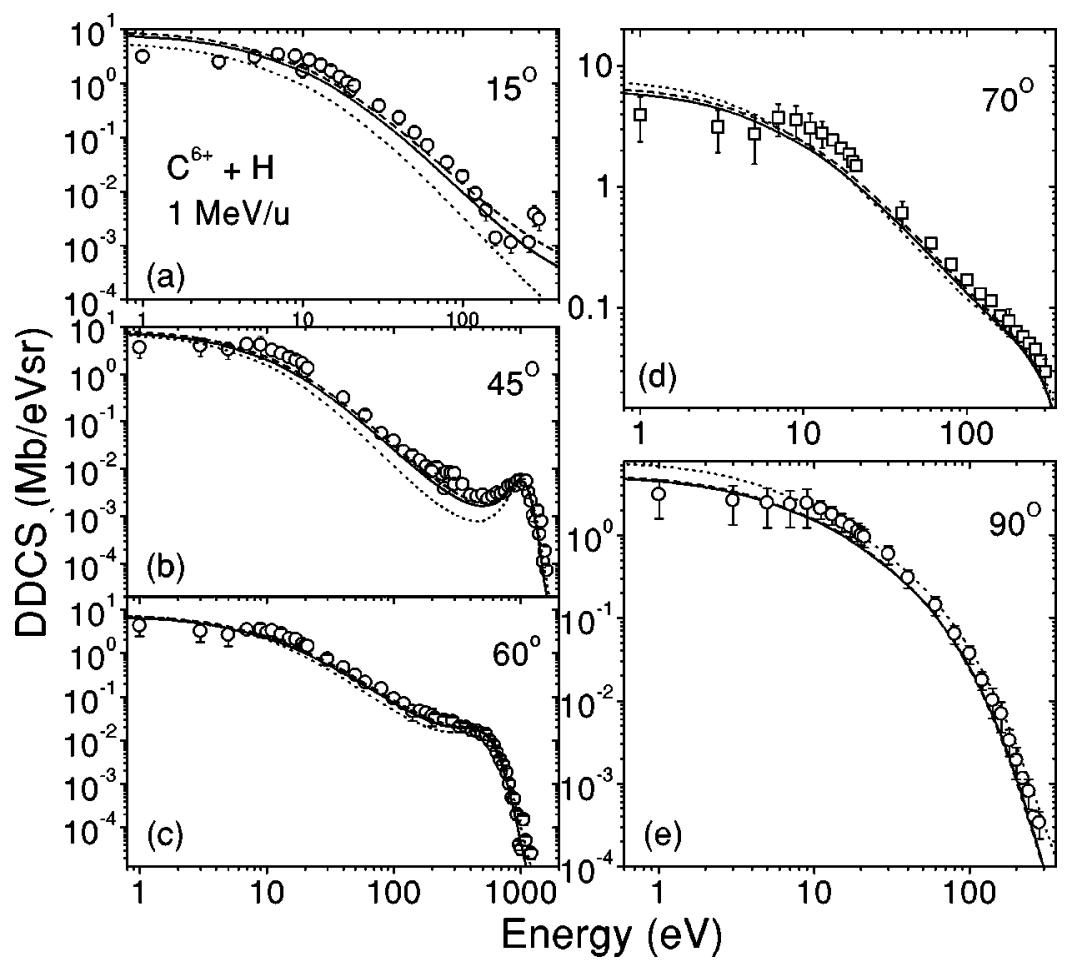

FIG. 3. (a) The electron energy distributions of electron DDCS for different emission angles in forward direction. The CDW-EIS, CDW, and $B 1$ calculations are shown by solid, dashed, and dotted lines, respectively.

model gives a much better agreement compared to the $B 1$ model, but still underestimates the data by almost a factor of 2 at extreme forward angles. The CDW calculations give a good agreement with the data over the whole angular range at these energies. The differences between the CDW-EIS and CDW models are observed only for small forward and large backward angles and the difference remains small (within about 20\%) below $50 \mathrm{eV}$. Above $100 \mathrm{eV}$ [see Figs. 2(d)2(f)] the CDW values are a factor of 2 to 4 higher than the CDW-EIS values at backward angles. The experimental data seem to support the CDW values for backward emissions. The emission cross sections in the transverse cone, i.e., between $60^{\circ}$ and $120^{\circ}$, are very well reproduced by both of the distorted-wave models.

\section{B. Energy distributions of DDCSs}

The energy distributions of DDCSs measured at the forward angles $\theta=15^{\circ}, 45^{\circ}, 60^{\circ}, 70^{\circ}$, and $90^{\circ}$ are shown in Fig. 3. For $\theta=15^{\circ}$, the general agreement with the CDWEIS and CDW models is quite good. However, these models give slightly lower cross sections above $10 \mathrm{eV}$. At this extreme forward angle, the $B 1$ calculations fall well below the data throughout most of the energy range. At $45^{\circ}$, the agreement with the theories is similar to the case of $15^{\circ}$ except that now all the theories come closer to the data and the $B 1$ results still fall well below the DDCS data between $5 \mathrm{eV}$ and the binary encounter (BE) peak position. For $\theta=60^{\circ}-90^{\circ}$, all the calculations, however, provide quite good agreement with the data.

As seen in Fig. 4, at $105^{\circ}$ the distorted-wave models are in good agreement with the data while the $B 1$ calculation yields cross sections that are larger by a factor of 2-3. At larger angles, both distorted-wave calculations give good agreement with the data below $100 \mathrm{eV}$ but at higher energies the CDW-EIS calculations fall below the data while the CDW calculation remains in good agreement. At $160^{\circ}$, the CDW results fall above the CDW-EIS results at even lower energies, and agree better with the data. A good agreement between the CDW model and the data may indicate that the TCE is described better by this model compared to the CDW-EIS model.

\section{Forward-backward asymmetry}

It is obvious from the previous discussions that the $B 1$ calculations underestimate the DDCS data in the extreme forward angles and overestimate them for large backward angles. Such a forward-backward asymmetry is caused by the two-center effect, which will be clear in Fig. 5, in which we show the ratio $(R)$ of the DDCS data to the $B 1$ calculations. The deviation of $R$ from 1.0 can be directly interpreted as the quantitative estimate of the two-center effect. For example, in the forward angles the ratio increases with electron energy [Figs. 5(a)-5(c)] since electrons with gradually increasing energies correspond to those produced at smaller impact parameter collisions. The relative velocity between the electrons and the projectile ions is decreasing and hence is subjected to larger two-center effects. The ratio peaks at an electron energy that approximately corresponds to electron velocity $v_{e}=v_{p} \cos \theta$, where $v_{p}$ is the cusp electron velocity, which is the same as the velocity of the projectile. The energies of these electrons, i.e., $\varepsilon=E_{p}\left(m / M_{p}\right) \cos ^{2} \theta$ $=548.58\left(E_{p} / M_{p}\right) \cos ^{2} \theta \mathrm{eV}$ (where $E_{p}$ and $M_{p}$ are the energy and mass of the projectile, $m$ being the electron mass), are indicated by arrows in Figs. 5(a)-5(c). It is known that at zero degree the cusp electrons are produced as a result of projectile interaction with the electrons moving with velocity $v_{p}$, which is not included in the $B 1$ calculations. At higher energies [i.e., $E_{e} \geqslant E_{p}(m / M) \cos ^{2} \theta$ ], there is a greater differ- 


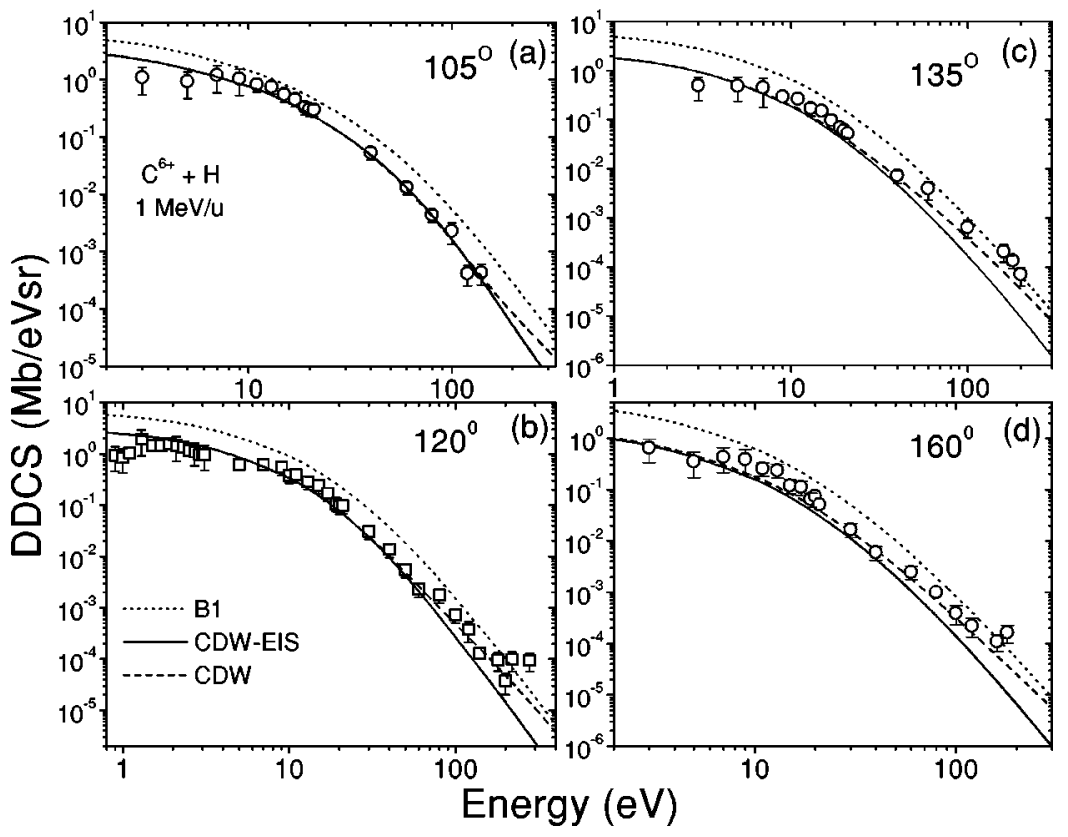

FIG. 4. Energy distributions of electron DDCS for different backward angles. The lines have the same meaning as in Fig. 3. ence between the electron and the projectile velocities and hence the interaction between the electrons and projectiles is again reduced. The minimum is obtained at the BE peak position.

The ratio is much less than 1.0 for backward angles, indicating the inadequacy of the $B 1$ calculations in predicting

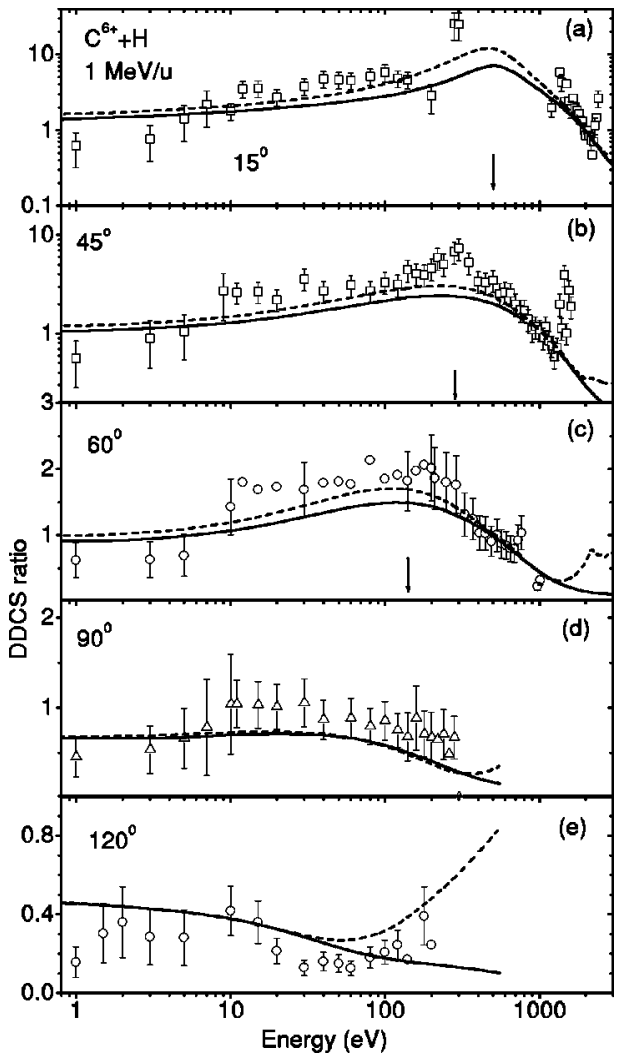

FIG. 5. The DDCS ratio, i.e., the DDCS data divided by the $B 1$ calculations, along with the corresponding ratios of the CDW-EIS and CDW to the $B 1$ calculations. The different panels display the ratios for different emission angles. the electron emission in large backward directions. The backward emission could also be affected by a double collision [30], i.e., the electrons that are liberated by the interaction with the passing projectile are further scattered from the target atom (or recoil ion). The CDW calculations show a slightly better agreement with the data compared to the CDW-EIS model. This shows that a more detailed description of the ionization mechanism cannot be accounted for without considering the electron as moving in a two-center field created by the heavy particles in the entire time of collision. In the CDW-EIS model, as is mentioned in the Introduction, this two-center character is emphasized mostly in the outgoing channel. So the above results show that better agreement and finer details on the DDCS can only be achieved by including the two-center dynamics of electrons also in the incoming path of the collision, as is done in the CDW model.

\section{Single differential and total cross sections}

The single differential distributions $d \sigma / d \Omega$ were derived by performing numerical integration of the energy distributions of the DDCS and are shown in Fig. 6 (see also Table II). The distribution has a peaklike structure that is highly asymmetric about the peak position, i.e., a large forwardbackward asymmetry is obvious. The $B 1$ calculations, in contrast, predict a symmetric distribution near the peak position. The continuum distorted-wave calculations, on the other hand, reproduce the distribution quite well, giving a large forward-backward asymmetry caused by the two-center effect. The CDW-EIS calculations are slightly lower in the forward angles compared to the CDW calculations. The data do not allow us to differentiate between the two calculations.

The total cross sections were calculated by integrating the single differential cross section (SDCS) in Fig. 6. The experimental total cross section is found to be $622 \mathrm{Mb}$, which is in excellent agreement with the CDW (633 Mb) and CDW-EIS $(560 \mathrm{Mb})$ predictions. The $B 1$ calculations are 


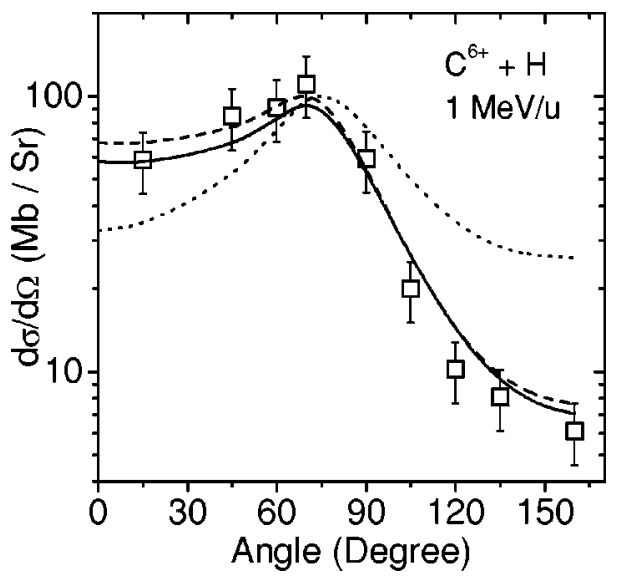

FIG. 6. The energy integrated angular distributions $d \sigma / d \Omega$ for $12 \mathrm{MeV} \mathrm{C}^{6+}+\mathrm{H}$. The data are compared to the $B 1, \mathrm{CDW}-\mathrm{EIS}$, and the CDW calculations using the different lines described in Fig. 2.

within $10 \%$ of the measured data. Therefore, the total cross sections are not a very sensitive test of these different theoretical models, since all of them agree with the data within about $10 \%$, whereas large deviations are observed in the case of DDCS data and therefore they provide a more sensitive test. The total cross sections mentioned above include electrons having energies between 1 and $300 \mathrm{eV}$ and are emitted between $15^{\circ}$ and $180^{\circ}$. The electrons outside those ranges will increase the cross sections, but only by a few percent.

\section{CONCLUSIONS}

We have measured the double differential cross sections of low-energy $(1-300 \mathrm{eV})$ electron emission for a pure threebody collision system involving a bare ion on an atomic hydrogen target. The two-center effect has been explored in detail from the observed forward-backward asymmetry by measuring the angular distribution of these electrons. The $B 1$ calculations give much lower DDCS at small forward angles,
TABLE II. Single differential cross sections $(d \sigma / d \Omega)$ for different angles in units of $\mathrm{Mb} / \mathrm{sr}$. Typical errors are about $25 \%$.

\begin{tabular}{cccccccccc}
\hline \hline $15^{\circ}$ & $30^{\circ}$ & $45^{\circ}$ & $60^{\circ}$ & $70^{\circ}$ & $90^{\circ}$ & $105^{\circ}$ & $120^{\circ}$ & $135^{\circ}$ & $160^{\circ}$ \\
\hline 58.8 & 28 & 84.6 & 90.9 & 110.9 & 59.4 & 20.0 & 10.2 & 8.1 & 6.1 \\
\hline \hline
\end{tabular}

much higher values at large backward angles, and provide a good agreement for angles around $90^{\circ}$. The CDW-EIS and CDW models provide a reasonable agreement for all the angles and energy ranges, although some discrepancies exist for electron emission in extreme forward and backward angles. The CDW model predicts slightly higher cross sections than the CDW-EIS model and agrees better with the data at higher energies. The finer details of the two-center electron emission are better represented by the CDW model compared to the CDW-EIS model. The single differential distributions are also studied and a large forward-backward asymmetry is observed, which is reproduced very well by the continuum distorted wave calculations. The $B 1$ calculations fail completely to predict the observed large asymmetry in the DDCS and SDCS. The total cross sections derived by integrating the measured data are, however, in good agreement (i.e., within 10\%) with all the models studied here. The two-center effect among the collision partners in the final state plays an important role in the electron emission in the present collision system with velocity $v_{p}=6.35$ a.u. and perturbation strength $Z_{p} / v_{p} \sim 1$.

\section{ACKNOWLEDGMENTS}

This work was supported by the Division of Chemical Sciences, Office of Basic Energy Sciences, Geosciences and Biosciences, Office of Science, U.S. Department of Energy. One of us (L.C.T.) would like to gratefully acknowledge the support provided by the JRM laboratory, KSU during this work, and L.G. gratefully acknowledges a grant from the J. Bolyai Research Fund.
[1] W.L. Fite, R. Stebbings, D.G. Hummer, and R.T. Brackmann, Phys. Rev. 119, 663 (1960).

[2] M.B. Shah and H.B. Gilbody, J. Phys. B 14, 2361 (1981).

[3] M.B. Shah, D.S. Elliot, and H.B. Gilbody, J. Phys. B 20, 2481 (1987)

[4] T.W. Shyn, Phys. Rev. A 45, 2951 (1992).

[5] M.W. Gealy, G.W. Kerby III, Y.-Y. Hsu, and M.E. Rudd, Phys. Rev. A 51, 2247 (1995).

[6] G.W. Kerby III, M.W. Gealy, Y.-Y. Hsu, and M.E. Rudd, Phys. Rev. A 51, 2256 (1995).

[7] Y.-Y. Hsu, M.W. Gealy, G.W. Kerby III, and M.E. Rudd, Phys. Rev. A 53, 297 (1996); Y.-Y. Hsu, M.W. Gealy, G.W. Kerby III, M.E. Rudd, D.R. Schultz, and C.O. Reinhold, ibid. 53, 303 (1996).

[8] Lokesh C. Tribedi, P. Richard, W. DeHaven, L. Gulyás, and M.E. Rudd, J. Phys. B 31, L369 (1998).

[9] N. Stolterfoht, H. Platten, G. Schiwietz, D. Schneider, L. Gu- lyás, P.D. Fainstein, and A. Salin, Phys. Rev. A 52, 3796 (1995).

[10] D.S.F. Crothers and J.F. McCann, J. Phys. B 16, 3229 (1983).

[11] P.D. Fainstein, V.H. Ponce, and R.D. Rivarola, J. Phys. B 21, 287 (1988).

[12] P.D. Fainstein, V.H. Ponce, and R.D. Rivarola, J. Phys. B 24, 3091 (1991).

[13] Dź Belkic, J. Phys. B 11, 3529 (1978).

[14] M. Brauner and J. H. Macek, Phys. Rev. A 46, 2519 (1992).

[15] L. Gulyás, P.D. Fainstein, and A. Salin, J. Phys. B 28, 245 (1995).

[16] L. Gulyás and P.D. Fainstein, J. Phys. B 31, 3297 (1998).

[17] T. Kirchner, L. Gulyás, H. J. Luedde, E. Engel, and R. M. Dreizler, Phys. Rev. A 58, 2063 (1998).

[18] R. Moshammer, J. Ullrich, M. Unverzagt, W. Schmidt, P. Jardin, R.E. Olson, R. Mann, R. Dörner, V. Mergel, U. Buck, and H. Schmidt-Böcking, Phys. Rev. Lett. 73, 3371 (1994). 
[19] Lokesh C. Tribedi, P. Richard, Y.D. Wang, C.D. Lin, and R.E. Olson, Phys. Rev. Lett. 77, 3767 (1996).

[20] Y.D. Wang, Lokesh C. Tribedi, P. Richard, C.L. Cocke, V.D. Rodríguez, and C.D. Lin, J. Phys. B 29, L203 (1996).

[21] Lokesh C. Tribedi, P. Richard, Y.D. Wang, C.D. Lin, R.E. Olson, and L. Gulyás, Phys. Rev. A 58, 3626 (1998).

[22] R.E. Olson, C.J. Wood, H. Schmidt-Böcking, R. Moshammer, and J. Ullrich, Phys. Rev. A 58, 270 (1998).

[23] Lokesh C. Tribedi, P. Richard, Y.D. Wang, C.D. Lin, L. Gulyás, and M.E. Rudd, Phys. Rev. A 58, 3626 (1998).

[24] J. Slevin and W. Sterling, Rev. Sci. Instrum. 52, 1780 (1981).

[25] M.E. Rudd, L.H. Toburen, and N. Stolterfoht, At. Data Nucl.
Data Tables 18, 413 (1976).

[26] L.H. Toburen, Phys. Rev. A 3, 216 (1971).

[27] M.A. Bolorizadeh and M.E. Rudd, Phys. Rev. A 33, 882 (1986).

[28] V.V. Afrosimov, G.A. Leiko, Y.A. Mamaev, and M.N. Panov, Zh. Éksp. Teor. Fiz. 56 (1969) [Sov. Phys. JETP 29, 649 (1969)].

[29] R.M. Wood, A.K. Edwards, and M.F. Steuer, Phys. Rev. A 15, 1433 (1977).

[30] N. Stolterfoht, R.D. DuBois, and R.D. Rivaola, Electron Emission in Heavy-Ion Atom Collision, Springer Series on Atoms and Plasmas (Springer, Berlin, 1997). 